\title{
Reticular thalamic responses to nociceptive inputs in anesthetized rats
}

\author{
Chen-Tung Yen ${ }^{\mathrm{a}, *}$, Fu-Zen Shaw ${ }^{\mathrm{b}}$ \\ ${ }^{a}$ Department of Zoology, National Taiwan University, \#1, Sect. 4, Roosevelt Road, Taipei, 106 Taiwan, ROC \\ ${ }^{\mathrm{b}}$ Institute of Neuroscience, Tzu Chi University, Hualien, Taiwan, ROC
}

Accepted 3 January 2003

\begin{abstract}
The present study compares nociceptive responses of neurons in the reticular thalamic nucleus (RT) to those of the ventroposterior lateral nucleus (VPL). Extracellular single-unit activities of cells in the RT and VPL were recorded in anesthetized rats. Only units with identified tactile receptive fields in the forepaw or hindpaw were studied. In the first series of experiments, RT and VPL responses to pinching with a small artery clamp were tested with the rats under pentobarbital, urethane, ketamine, or halothane anesthesia. Under all types of anesthesia, many RT units were inhibited. Second, the specificity of the nociceptive response was tested by pinching and noxious heating of the unit's tactile receptive field. Of the 39 VPL units tested, 20 were excited by both types of noxious stimuli. In sharp contrast, of the 30 RT units tested, none were excited and 17 were inhibited. In a third series of experiments, low-intensity and beam-diffused $\mathrm{CO}_{2}$ laser irradiation was used to activate peripheral nociceptive afferents. Wide-dynamic-range VPL units responded with short- and long-latency excitations. In contrast, RT units had short-latency excitation followed by long-latency inhibition. Nociceptive input inhibited RT units in less than $500 \mathrm{~ms}$. We conclude that a significant portion of RT neurons were polysynaptically inhibited by nociceptive inputs. Since all the cells tested were excited by light tactile inputs, the somatosensory RT may serve in the role of a modality gate, which modifies (i.e. inhibits) tactile inputs while letting noxious inputs pass.
\end{abstract}

(C) 2003 Elsevier Science B.V. All rights reserved.

Theme: Sensory system

Topic: Pain pathway

Keywords: Nociception; Pain; Pentobarbital; Thalamic reticular nucleus; Thalamus; Urethane; Ventroposterior lateral nucleus

\section{Introduction}

Several pathways have been proposed for the conduction of peripheral nociceptive information to pain-related higher centers. For example, the spinothalamic pathway is the most important pathway for transmission of discriminative aspects of nociceptive activity to the cortex via thalamocortical neurons (the lateral thalamic pathway; $[6,46])$. At least two major negative-feedback modulatory circuits have been described to control/modulate sensory information sent through this ascending pathway: the medullospinal and the reticulo-thalamocortical networks. The medullospinal descending circuitry acts principally on neuronal activities in the superficial laminae of the spinal

*Corresponding author. Tel.: +886-2-2363-0231x3322; fax: +886-22363-6837.

E-mail address: ctyen@ntu.edu.tw (C.T. Yen). cord [12]. In contrast, very few reports studied reticulothalamocortical modulation of the ascending nociceptive information [29,33].

The reticular nucleus of the thalamus (RT) is a thin layer of neurons covering the rostral, lateral, and dorsal parts of the thalamus. Ascending thalamocortical and descending corticothalamic fibers make collateral branches here and terminate heavily [22]. RT neurons, in turn, send inhibitory output to the thalamic nuclei $[22,48]$. This sets up an exquisitely interconnected neural web between the cortex, thalamus and RT.

The RT has been implicated in various functions. Among these are attention [18,30], generation of brain waves [45] and modulation of sensory transmission $[24,25,45]$. In rats, the somatosensory part of the RT may have a more important role in the processing of sensory information because the ventrobasal nuclei (VB), including the ventroposterior medial (VPM) and ventroposterior 
lateral (VPL) nuclei, have few intrinsic GABAergic interneurons themselves [1]. Therefore, processing of somatosensory information at the thalamic level in the lateral thalamic pathway of the rat depends almost exclusively on thalamocortical neurons and RT neurons. Previous studies have shown that the receptive field and neuronal response of VPM neurons to innocuous stimuli can be regulated by GABAergic RT neurons [24,25]. Also, there have been many papers examining the nociceptive processing of VPL neurons (in rats: [15-17,27,29,34,50]). In contrast, there have been very few studies of the responses of RT neurons to noxious stimulation [29,33]. These few studies were inconclusive because either the functions of the recorded RT neurons were unknown [33], or the somatosensory receptive field of the RT neurons were scattered over the head and body [29], thus, specific noxious stimuli could not be applied. The present study was performed to examine systematically the responses of the RT neurons in the somatosensory sector to noxious inputs.

To be able to use more specific noxious stimuli, we recorded only from RT neurons whose tactile receptive fields are located in the glabrous skin of the forepaw or hindpaw. Mechanical noxious stimuli were applied with an arterial clamp. A servo-controlled Peltier device and a $\mathrm{CO}_{2}$ laser stimulator were used to deliver specific noxious thermal stimuli. With the use of rapid, synchronized laser thermal irradiation, the time course of excitation and inhibition can be precisely determined. Corresponding VPL neurons were tested as a positive control, and their responsive patterns were compared. The studies were performed on anesthetized rats. To be able to obtain a more general conclusion, four types of anesthetics, i.e. pentobarbital, urethane, ketamine and halothane, were used. This work has been reported in preliminary form [47].

\section{Materials and methods}

Forty-eight adult Long Evans rats, weighing 250-00 g, were used. Four kinds of anesthesia regimens were used. These were sodium pentobarbital (i.p., $50 \mathrm{mg} / \mathrm{kg}$ initially, thirty-three rats), urethane (i.p., $1300 \mathrm{mg} / \mathrm{kg}$ initially, nine rats), ketamine (i.p., $100 \mathrm{mg} / \mathrm{kg}$ initially, four rats), and halothane $(5 \%$ in oxygen initially, with maintenance at $1 \%$, two rats). The anesthetic level of the rats was continuously monitored with arterial blood pressure, heart rate, and EEG recordings. Supplementary doses of diluted anesthetics of the same kind were given as needed such that the rats were atonic with no spontaneous movement throughout the experiment. The flexor reflexes of the rats were kept to a minimum. The entire surgical and experiment protocol complied with NIH (USA) recommended procedures for animal use and care and was approved by the Experimental Animal Committee of Tzu Chi University.
The femoral artery and vein on the right side and trachea were cannulated. Rectal temperature of the rat was kept between 37 and $38^{\circ} \mathrm{C}$ with a servo-controlled heating pad. The rat was mounted on a stereotaxic apparatus. A craniotomy was performed on the right parietal bone. Extracellular single-unit activities of neurons in the right RT and VPL were recorded with parylene insulated tungsten microelectrodes $(0.005$ inch diameter, $5 \mathrm{M} \Omega$ impedence, cat. no.: 573400, AM systems, Carlsborg). Two stainless steel screws were implanted into the frontal bones, one on each side. These screws served as EEG recording leads and as the reference electrode for the single-unit recording. Neural signals were amplified with conventional amplifier and monitored visually and aurally. Signals were also displayed on a fast chart recorder (Gould TA11) and fed into a data acquisition system (MAP, Plexon, Dallas, TX, USA). Single unit data were captured and analyzed with SORTCLIENT, OFFLINE SORTER and NEX programs (Plexon). Neural signals were also stored on tape for later analysis (DR890, Neuro Data Instruments). To assure uniform, high quality recording, the metal microelectrodes were not reused after a marking DC lesion had been performed. Special care was also taken to collect only clearly isolated single-unit data.

After isolation of a single-unit in the thalamus, a battery of somatosensory stimuli was tested. These included innocuous stimuli, such as air puffs, tapping, pressing, and brushing of body parts, and noxious ones, i.e. pinching with a small artery clamp (contact area $2 \mathrm{~mm}$ wide, less than $8 \mathrm{~mm}$ long) or a $\mathrm{CO}_{2}$ laser whose beam was diffused to $3 \mathrm{~mm}$ in diameter $[38,39,51]$. Another test was a controlled temperature increase of the contacted skin surface with a hand-held Peltier device. The contact region of the Peltier device had a surface area of $25 \mathrm{~mm}^{2}$, which was made of copper. The temperature of the contact region was measured with a thermocouple glued to its surface. The basal temperature of the contact region was set at $40{ }^{\circ} \mathrm{C}$; temperature steps were feedback-controlled to increase to a final temperature of $55^{\circ} \mathrm{C}$ with an initial rising rate of $1{ }^{\circ} \mathrm{C} / \mathrm{s}$. The total duration of the temperature change was set to a fixed value of $30 \mathrm{~s}$. The rising rate of the thermal probe leveled off as the target temperature got nearer, such that the probe temperature was above $50{ }^{\circ} \mathrm{C}$ for a duration of about $10 \mathrm{~s}$.

To test whether a feedback loop from the primary somatosensory cortex (SI) was involved in nociceptive RT inhibition, the ipsilateral SI was removed in a few rats under pentobarbital anesthesia. This was done by suctioning away the cerebral cortex from $2 \mathrm{~mm}$ rostral to $4 \mathrm{~mm}$ caudal to the bregma anteroposteriorly, and from the midline to $5 \mathrm{~mm}$ to the right of the midline mediolaterally. To minimize cerebral edema, five of the rats were paralyzed with gallamine triethiodide $(2 \mathrm{mg} / \mathrm{kg}$, initially, and $1 \mathrm{mg} / \mathrm{kg} / \mathrm{h}$ subsequently) and ventilated through a trachea cannula with a rodent ventilator. Expiratory $\mathrm{CO}_{2}$ was monitored, and the end-tidal expiratory $\mathrm{CO}_{2}$ con- 
centration was kept between 3.5 and $4.5 \%$ by adjusting the volume and rate of the ventilator.

After the recording session, the rat was deeply anesthetized and intracardially perfused with a $4 \%$ formaldehyde solution. The brain was removed and placed in a $30 \%$ sucrose solution. Several days later, $100-\mu \mathrm{m}$ thick frozen sections were made serially with a sliding microtome. The sections were stained with cresyl violet. Recording loci were identified with track marks and selective lesion points made during the experiment (50-100 $\mu \mathrm{A}$ anodal DC current, $15 \mathrm{~s}$ ).

\section{Results}

\subsection{Many RT neurons were inhibited by pinching under pentobarbital, urethane, ketamine, and halothane}

RT units were identified by their characteristic bursts or tonic spontaneous activity [10, Fig. 1B], their long train of responses to peripheral stimulation [42, Fig. 2C1], and their location in the histological sections. Examples are shown in Figs. 1 and 2. Extracellularly recorded RT units had biphasic or triphasic waveforms with a prominent negative phase (Fig. 1C). The duration of the spike was shorter than those of the VPL units, in a range of $0.8-1.2$ ms. A characteristic rebound oscillation was elicited by electrical stimulation of the peripheral receptive field (Fig. 2C). In topographic organization, hindpaw RT units were found dorsal to the forepaw RT units, and several forepaw
RT units were found in the ventral-most location beneath the lower jaw RT units [41]. Units that had tactile fields in the paws were tested with mechanical stimuli. Examples of RT unit responses to cutaneous stimuli are illustrated in Fig. 3. Fig. 3A, B and $\mathrm{C}$ shows $\mathrm{RT}$ units recorded in rats under pentobarbital, urethane, and ketamine anesthesia, respectively. Light tapping with a small von Frey hair (Stoelting model 18011, about $5 \mathrm{~g}$, applied during the periods marked with horizontal bars) excited the RT units. In contrast, pinching the skin or digit within the receptive field with a small artery clamp (arrows) caused a cessation of the bursts of the spike waveform (Fig. 3A) or tonic activities (Fig. 3B and C) of the RT units.

Twenty-nine, sixteen, nine and two RT units were tested with the same pinch stimulus in rats anesthetized with pentobarbital, urethane, ketamine, and halothane, respectively. Of these, eighteen (62\%), seven (44\%), two (22\%), and one $(50 \%)$ were inhibited. Excitation was found in three $(10 \%)$ pentobarbital-anesthetized and one $(6.3 \%)$ urethane-anesthetized RT units. Activity of the other tactile RT units did not change with pinching.

VPL units, recorded extracellularly, had a biphasic waveform, about $1.5 \mathrm{~ms}$ in duration, with a prominent initial negativity (Fig. 1F). VPL units whose receptive fields were in the paws were isolated and tested. An example is illustrated in Fig. 4. A prominent short-latency peak was observed by electrical stimulation of the receptive field (5th digit of the left forepaw). Thirty-three, eighteen and twelve VPL units were tested with the pinching stimuli produced by the small arterial clamp
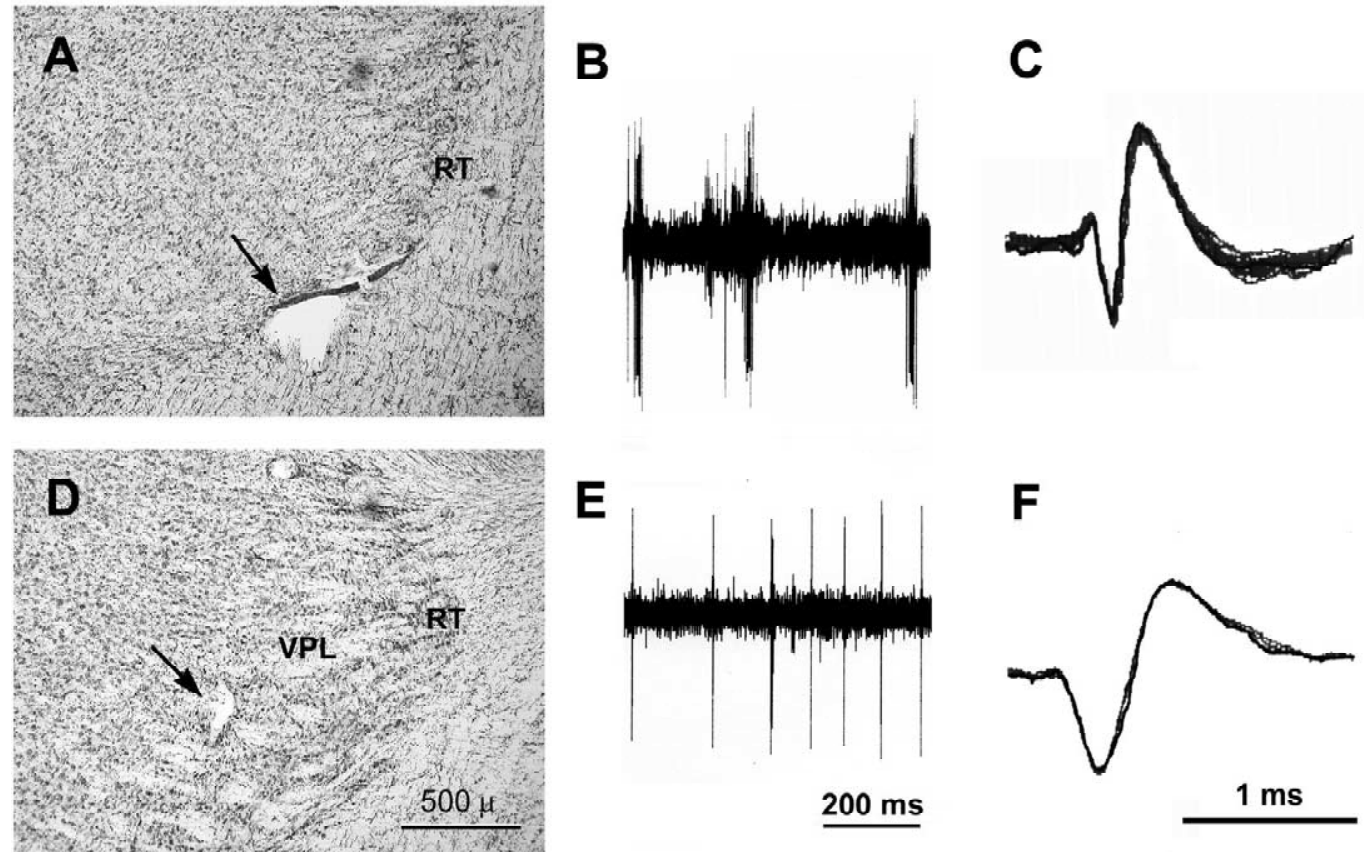

Fig. 1. Representative example of the criteria used to distinguish a RT unit. (A) The location of the recording was in the RT. The arrow points to the electrolytic lesion made at the recording site. This and (D) are photomicrographs of coronal section of the right thalamus. Medial is to the left. (B) Characteristic bursty spontaneous activity, and the triphasic spike waveform (C). (A), (B) and (C) were from the same RT unit. Corresponding properties of a VPL unit is illustrated in (D), (E) and (F). Note the longer and biphasic shape of the VPL spike waveform (F). Bandpass filter set at 300 to 3 KHz. 

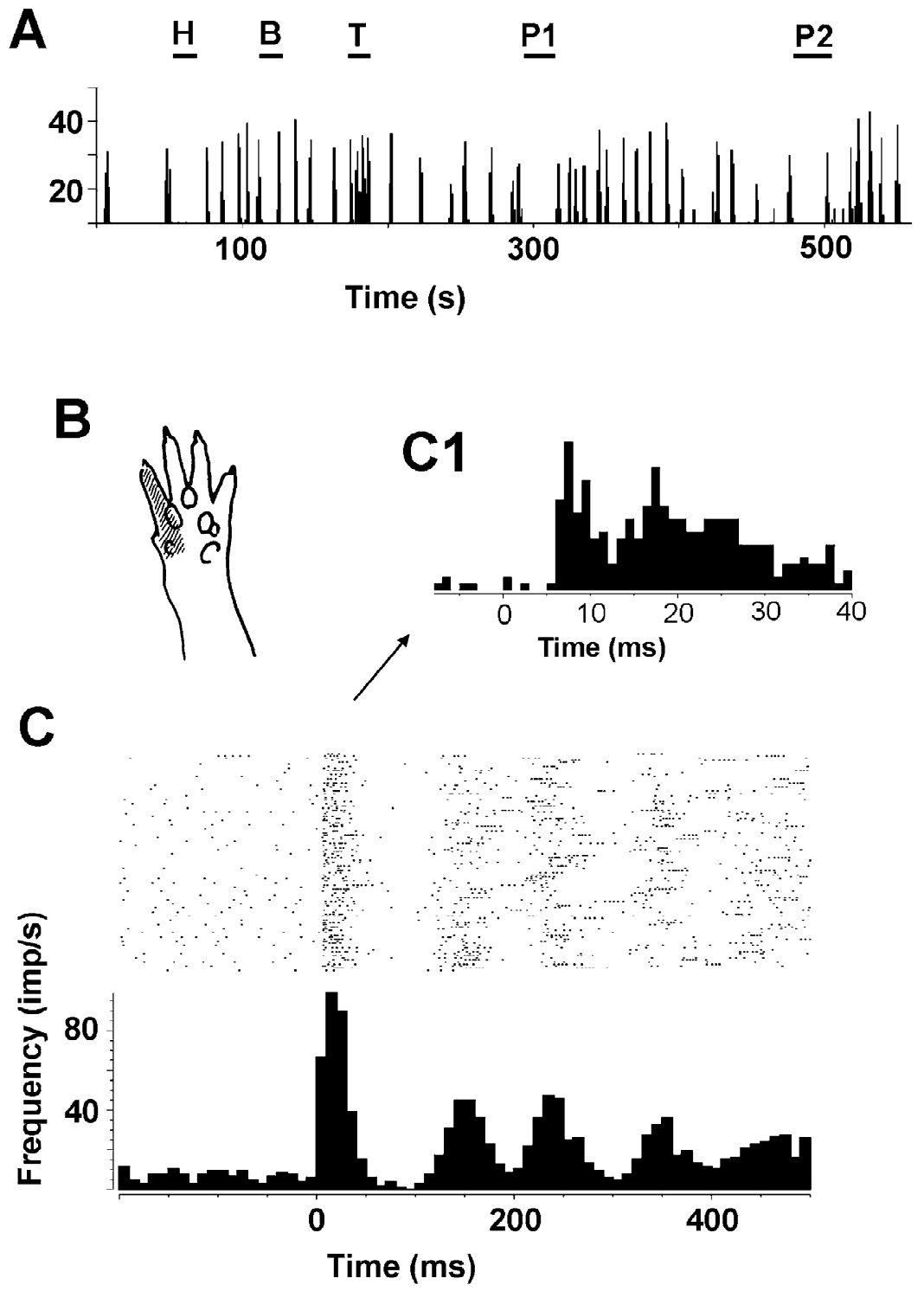

Fig. 2. Response properties of a representative RT unit under pentobarbital anesthesia. This unit had bursts of spontaneous activity. It was excited by light tapping (T) of the 1st and 2nd digits of the left forepaw (shaded area in B). Air puffs which moved only hairs (H) and brushing of the skin with a soft paint brush (B) produced no response. Pinching of the 2nd digit (P1) or the paw pad (P2) with a small arterial clamp caused cessation of spontaneous activity. Bin size: $1 \mathrm{~s}$. (C) Peri-event raster and histogram of 100 trials using current stimulation of $1 \mathrm{~Hz}, 2 \mathrm{~ms}, 400 \mu \mathrm{A}$ through a pair of needles inserted into the paw pads. The first peak of the response occurred about $7 \mathrm{~ms}$ as more clearly illustrated in the expanded histogram $\mathrm{C} 1$. Bin size is $10 \mathrm{~ms}$ for $\mathrm{C}$ and $1 \mathrm{~ms}$ for $\mathrm{C} 1$.

applied to their receptive fields in pentobarbital-, urethaneand ketamine-anesthetized rats, respectively. Twenty-four $(73 \%)$, six (33\%), and five (42\%) units were excited, respectively. These excitations were stronger than those elicited by the low-intensity stimuli (Fig. 4A). These units had similar characteristics of the wide-dynamic-range cells in the cat thalamus [21]. Eight (24\%), eleven (61\%), and seven $(58 \%)$ of the VPL units were not activated further with a pinching stimulus. These VPL units might be the low-threshold cells. Pinching inhibited 1 VPL unit each
(3\% and 6\%) in rats under pentobarbital and urethane anesthesia, respectively.

\subsection{Mechanical and thermal noxious stimuli had similar effects on the same RT neurons for both intact and SI- lesioned rats}

A servo-controlled contact thermal probe was used to test the specificity of the nociceptive response of the thalamic units. An example is illustrated in Fig. 5. Puffs of 
A

PB

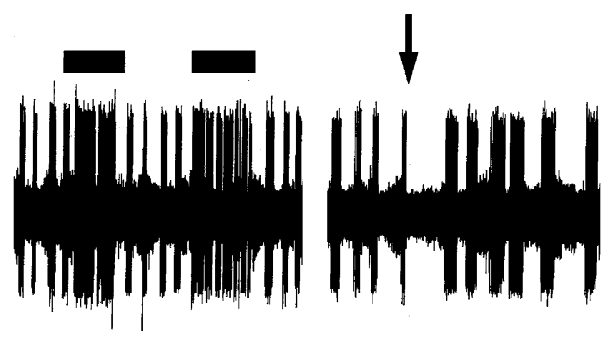

B
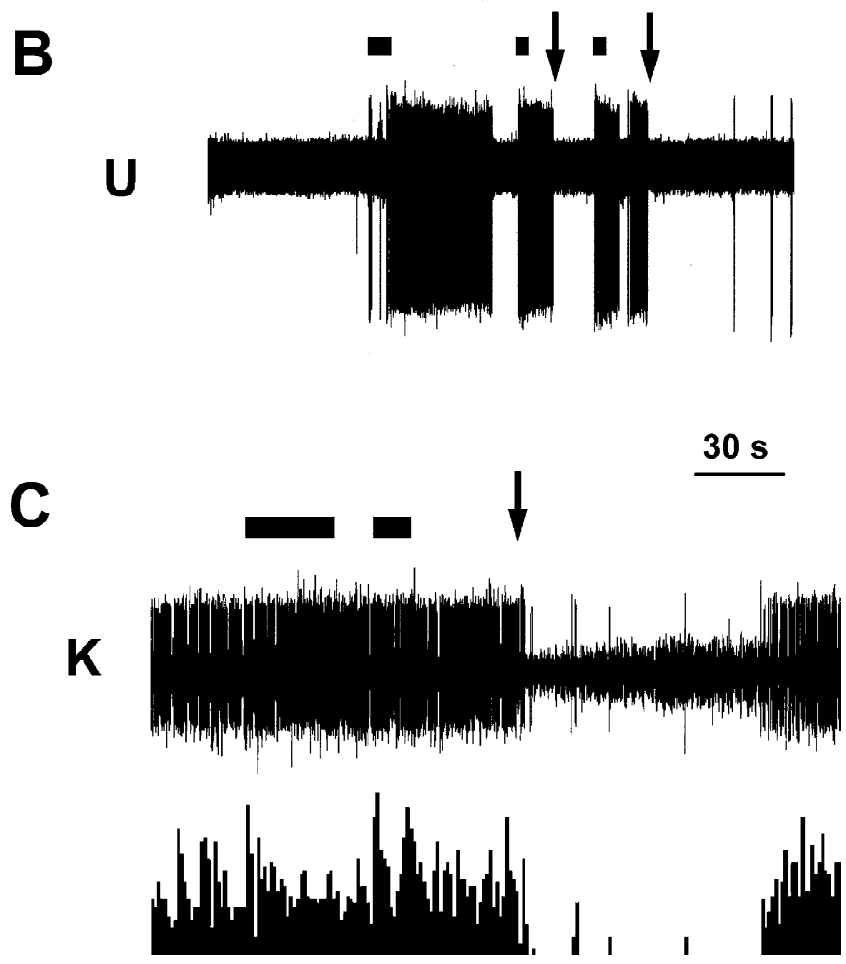

Fig. 3. Representative examples of RT responses to light tapping (horizontal bar) and pinching (arrow) under pentobarbital (PB), urethane $(\mathrm{U})$, or ketamine $(\mathrm{K})$ anesthesia. Note that RT units were excited by light tapping but inhibited by pinching. The lower panel in (C) is the histogram of the unit activity with a bin size of $1 \mathrm{~s}$. The same unit is used as the representative example shown in the top panel of the Fig. 1.

air or light tapping with a small von Frey hair of the 4th digit of the left forepaw excited this RT unit (Fig. 5A, light touch). In sharp contrast, brief pinching of the same digit with a small arterial clamp stopped its rhythmic firing (Fig. $3 \mathrm{~A}$, right panel). A rise in temperature of the thermal probe from 40 to $55^{\circ} \mathrm{C}$ also inhibited this unit (Fig. 5B).

Thirty RT units and thirty-nine VPL units were tested with both pinching (small artery clamp) and noxious heat (contact thermal probe). The distribution of the responsiveness pattern of these units is summarized in Table 1. A highly significant ( $\chi^{2}$ test, $P=0.0009$ ) correspondence of the results was found such that for the RT table, 22 (17 and $5,73 \%$ ) matched exactly, and none showed a mis- match (top right and bottom left corners of the $3 \times 3$ table). For the VPL table, the significance of the correspondence was even higher $(P<0.0001)$.

Ipsilateral sensorimotor decortication was performed in eleven rats under pentobarbital anesthesia. RT and VPL units were recorded and tested for the two noxious stimuli. The results are summarized in Table 2. Of the ten RT units recorded, $70 \%$ were found to be inhibited by both types of noxious stimuli, whereas $47 \%$ of the VPL units were excited. These values did not significantly differ from those in the intact group (Table 1).

\subsection{Laser irradiation excited wide-dynamic-range VPL neurons at $300 \mathrm{~ms}$ and inhibited RT neurons in less than $500 \mathrm{~ms}$}

The time course of the nociceptive excitation and inhibition was studied with rapid thermal stimuli produced by a $\mathrm{CO}_{2}$ laser. For better comparison, only units whose receptive fields were located in the glabrous skin of the left forepaw were studied. A pure inhibitory effect on a RT unit recorded in a pentobarbital-anesthetized rat is shown in Fig. 6A. The activity of this RT unit dropped to half the control value in $150 \mathrm{~ms}$. Total inhibition was reached within $200 \mathrm{~ms}$. In sharp contrast, a wide-dynamic-range VPL unit that had an overlapping receptive field recorded simultaneously through another microelectrode was strongly activated by the same laser irradiation $(11 \mathrm{~W}, 15 \mathrm{~ms})$ within a complementary time period from 300 to $1200 \mathrm{~ms}$ (Fig. 6B).

The $\mathrm{CO}_{2}$ laser produced more than simple nociceptive activation. The rapid increase in the cutaneous temperature caused a slight curling of the skin, which in a few cases activated some of the low-threshold receptors under the glabrous skin. An example is illustrated in Fig. 7. Laser pulses of $12 \mathrm{~W}$ and $10 \mathrm{~ms}$ excited this tonically active RT unit in a urethane-anesthetized rat at a short latency range (Fig. 7A and B). The excitation pattern and the latency of the RT unit's response to the low energy laser irradiation were comparable to those seen after low intensity electrical stimulation of the left forepaw (Fig. 7C). An increase in the laser energy to $13 \mathrm{~W}$ and $20 \mathrm{~ms}$ brought total cessation of the tonic spontaneous discharge for about $3 \mathrm{~s}$ within 500 ms (Fig. 6D). Threshold intensity of nocifensive behavioral responses to $\mathrm{CO}_{2}$ laser irradiation of the glabrous skin of the rat is about $0.09 \mathrm{~J}$ (hindpaw: [11]; tailbase: [51]), and A- $\beta$ response threshold of this RT unit to electrical stimulation was about $0.3 \mathrm{~mA}$.

Sixteen RT units and eleven VPL units recorded in twelve rats were tested with strong laser stimulation of their peripheral receptive fields in the forepaw region. The average responses are summarized in Fig. 8. A short latency excitation response was seen in five of the sixteen RT units (Fig. 8A, inset in the top panel). This short 

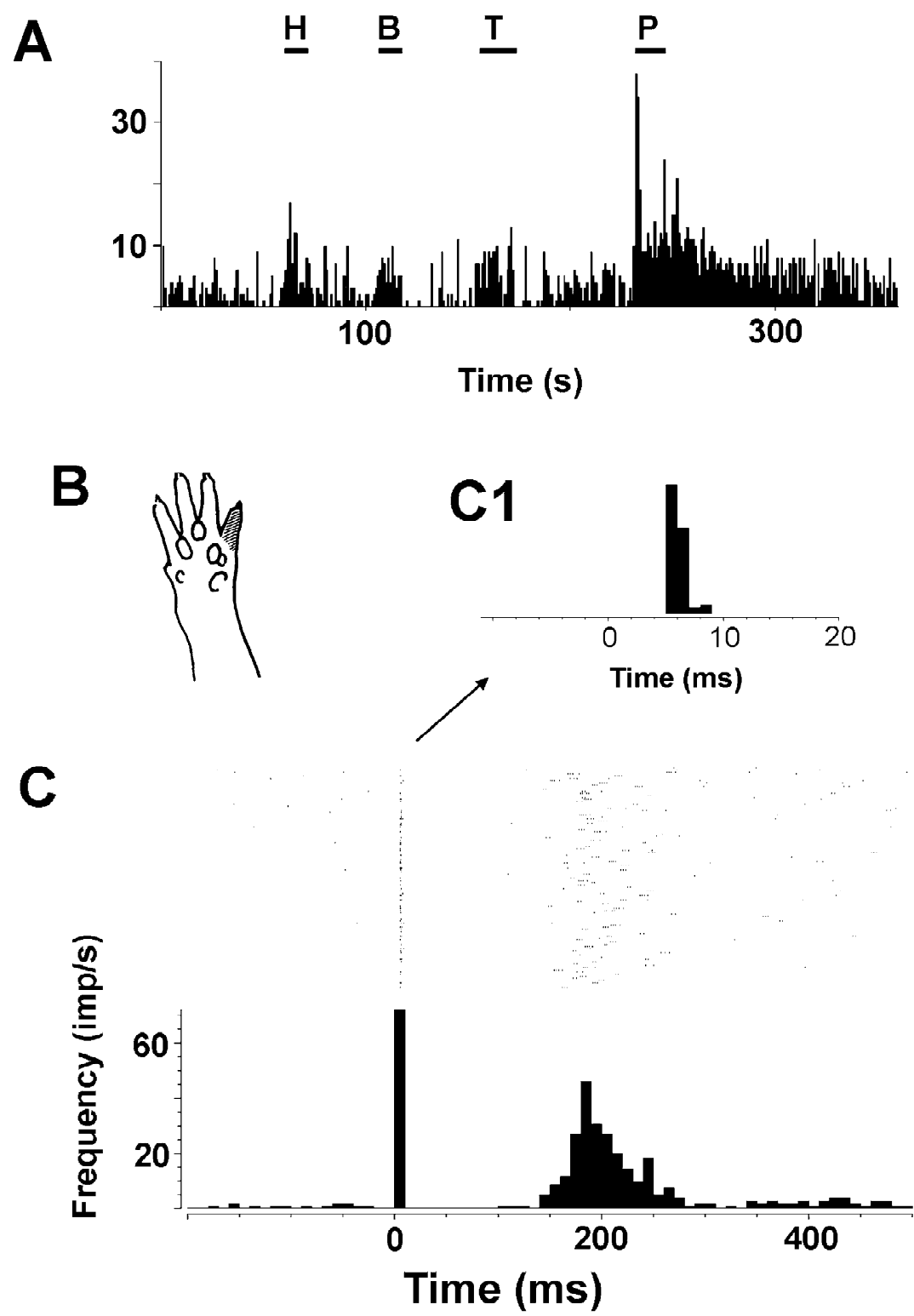

Fig. 4. Response properties of a representative wide-dynamic-range VPL unit under urethane anesthesia. (A) This unit responded to hair stimulation (H), brushing (B), and light tapping (T) of the 5th digit of the left forepaw (shaded area in B). However, the best response was seen when the 5th digit was pinched with a small arterial clamp (P). Bin size: $1 \mathrm{~s}$. (C) Peri-event raster and histogram of 100 trials using current stimulation of $1 \mathrm{~Hz}, 2 \mathrm{~ms}, 500 \mu \mathrm{A}$ through a pair of needles inserted into the paw pads under the 5th digit. The first peak of the response occurred at $5 \mathrm{~ms}$ as seen in the expanded histogram $\mathrm{C} 1$. Bin size is $10 \mathrm{~ms}$ for $\mathrm{C}$ and $1 \mathrm{~ms}$ for $\mathrm{C} 1$.

latency excitation began $10 \mathrm{~ms}$ after laser irradiation and lasted $50 \mathrm{~ms}$. There was a prominent inhibition period following this short latency excitation. Examining through individual units, the range of the half maximal inhibition latency varied between 150 and $700 \mathrm{~ms}$ (median: $450 \mathrm{~ms}$ ). Collectively, the average RT activity decreased below the 99\% confidence line $500 \mathrm{~ms}$ after laser irradiation. Minimal activity was reached $900 \mathrm{~ms}$ afterwards (Fig. 8A, lower panel). RT activity remained below the lower $99 \%$ confidence line for more than $3 \mathrm{~s}$.

The eleven wide-dynamic-range VPL units tested with laser irradiation, in contrast, showed two phases of excitation (Fig. 8A, top panel). Short excitation was observed in eight of the eleven units. It started at $5 \mathrm{~ms}$ and peaked 10 $\mathrm{ms}$ after laser irradiation. The duration of this excitation was short, at about $20 \mathrm{~ms}$. Nine of the VPL units showed the longer-latency excitation (open arrow in Fig. 8A). This long-latency excitation started at about $300 \mathrm{~ms}$ and peaked at $500 \mathrm{~ms}$. Significant excitation was observed for a duration of more than 2 s. In comparison, only shortlatency excitatory responses were observed in VPL and RT units following a light tapping tactile stimulus (Fig. 8B). 


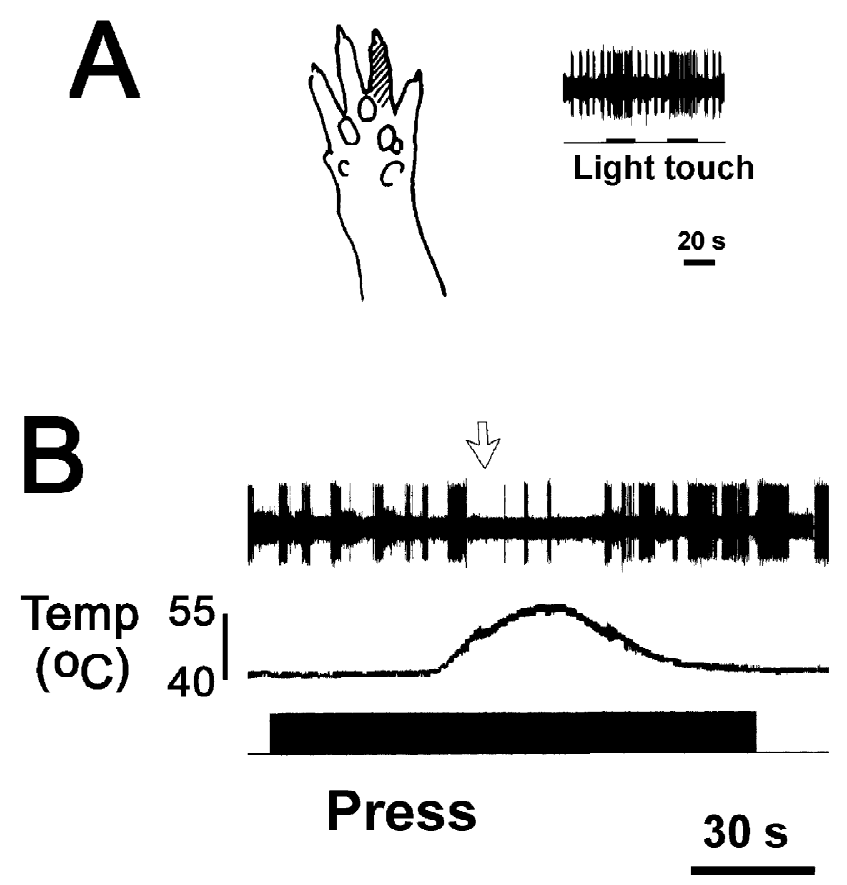

Fig. 5. RT unit excited by light touches of the 4th digit of the left forepaw (A). It was inhibited by mechanical pinching of the same digit (Fig. 3A), and by a rise in the skin temperature from 40 to $55^{\circ} \mathrm{C}$ (open arrow in B). The solid bar in the lowest panel marks the period at which the Peltier thermoprobe was pressed on the left palm covering the 4th digit (press).

\section{Discussion}

The present study brings three converging pieces of evidence together to show that a significant percentage of neurons in the RT are inhibited by noxious stimuli applied to their excitatory tactile receptive fields. Our first experiment showed that pinching inhibited many RT neurons in pentobarbital-, urethane-, ketamine- or halothane-anesthe-

Table 1

Distribution of the number of reticular thalamic (RT) or ventroposterior lateral (VPL) units which were either excited, inhibited, or not responsive to pinching or heating $\left(55^{\circ} \mathrm{C}\right)$ of their respective tactile receptive fields

\begin{tabular}{llllr}
\hline \multirow{2}{*}{ Heat } & \multicolumn{2}{l}{ Pinch } & & \\
\cline { 2 - 5 } RT & Excitation & No response & Inhibition & Total \\
\hline Excitation & 0 & 1 & 0 & 1 \\
No response & 3 & 5 & 1 & 9 \\
Inhibition & 0 & 3 & 17 & 20 \\
Total & 3 & 9 & 18 & 30 \\
VPL & & & & \\
Excitation & 20 & 0 & 0 & 20 \\
No response & 2 & 12 & 0 & 14 \\
Inhibition & 3 & 1 & 1 & 5 \\
Total & 25 & 13 & 1 & 39 \\
\hline
\end{tabular}

Table 2

Distribution of the number of reticular thalamic (RT) or ventroposterior lateral (VPL) units which were either excited, inhibited, or not responsive to pinching or heating $\left(55^{\circ} \mathrm{C}\right)$ of their respective tactile receptive fields in sensorimotor cortex-lesioned, pentobarbital-anesthetized rats

\begin{tabular}{llllc}
\hline & \multicolumn{2}{l}{ Pinch } & & \\
\cline { 2 - 5 } Heat & Excitation & No response & Inhibition & Total \\
\hline RT & & 0 & 0 & 0 \\
Excitation & 0 & 1 & 0 & 1 \\
No response & 0 & 2 & 7 & 9 \\
Inhibition & 0 & 3 & 7 & 10 \\
Total & 0 & & & \\
VPL & & 0 & 0 & 8 \\
Excitation & 8 & 6 & 0 & 6 \\
No response & 0 & 2 & 0 & 3 \\
Inhibition & 1 & 8 & 0 & 17 \\
Total & 9 & & & \\
\hline
\end{tabular}

tized rats. The percentage of nociceptor-inhibited RT neurons was higher in pentobarbital-anesthetized than in urethane-anesthetized rats. Also, inhibition of RT units required stronger laser pulses under urethane anesthesia (Fig. 6). Thus, the inhibitory nociceptive influence on RT neurons might be potentiated in pentobarbital anesthesia. The second experiment showed that most of the RT neurons inhibited by pinching were also inhibited by contact noxious thermal stimulus. The third experiment utilized short-pulse laser irradiation as the noxious heat source. RT neurons responded to the laser heat pulses with a long-latency and long-lasting inhibitory component. This inhibition began at $500 \mathrm{~ms}$ on average, and the latency was compatible with a polysynaptic C-fiber afferent input $[38,39]$.

The thalamus is an important relay station for sensory information transmission from the peripheral sense organs to the cerebral cortex. Moreover, at this level, the information is not simply passed on, but can be actively gated $[44,45]$. Several lines of evidence have indicated that the most important modulator at the level of the thalamus for the processing of somatosensory information probably is the RT. First, the VB of the rat has few intrinsic GABAergic interneurons $[1,19]$, and the RT provides a dense GABAergic projection to the VB [45,48]. Second, the RT can alter the short- and long-latency evoked responses elicited by innocuous stimulation and the tactile receptive field of VB neurons [3,20,24,25,31]. Lastly, administering GABAergic agents in the thalamus has a more potent inhibitory effect on nociceptive signals than does the administration of an opioid [23,37]. In addition, we herein provide evidence that many RT neurons are inhibited by noxious stimulation (Figs. 3, 5 and 8, Table 1 ), and that VPL neuronal activity concurrently increases (Figs. 5B and 8, Table 1). All these data converge to the possibility that the RT plays an important role in processing ascending nociceptive sensory information. 
A
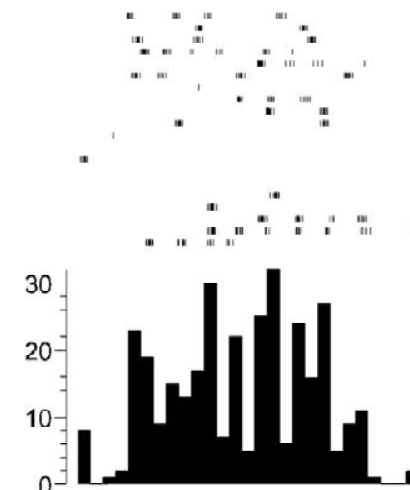

B
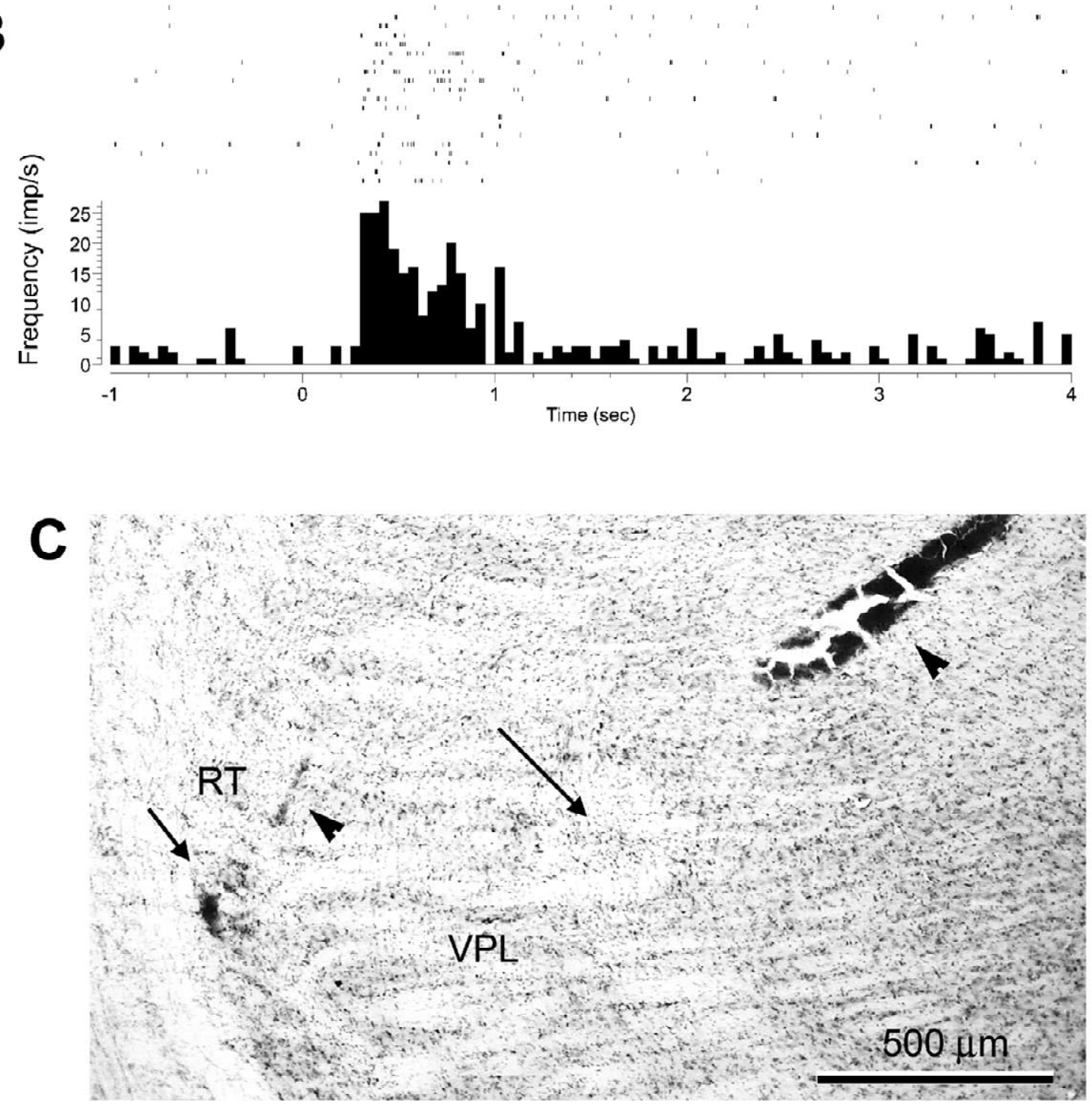

Fig. 6. (A) Example of dot raster (upper panel) and peri-event histogram (lower panel) of a RT unit inhibited by laser irradiation. This rat was under pentobarbital anesthesia. Note the bursty spontaneous activities of the RT neuron. The laser pulses were $11 \mathrm{~W}$ for $15 \mathrm{~ms}$ directed to the glabrous skin of the left forepaw at time 0. (B) In contrast, a VPL unit recorded simultaneously with a second microelectrode was strongly excited. Both neurons had a tactile receptive field within the left forepaw. Bin width: $50 \mathrm{~ms}$. (C) Photomicrograph of sagittally sectioned thalamus showing the two recording tracts (arrowheads), and the two lesions made at the recording sites (arrows). The longer arrow points to the VPL lesion. Rostral is to the left. 
A
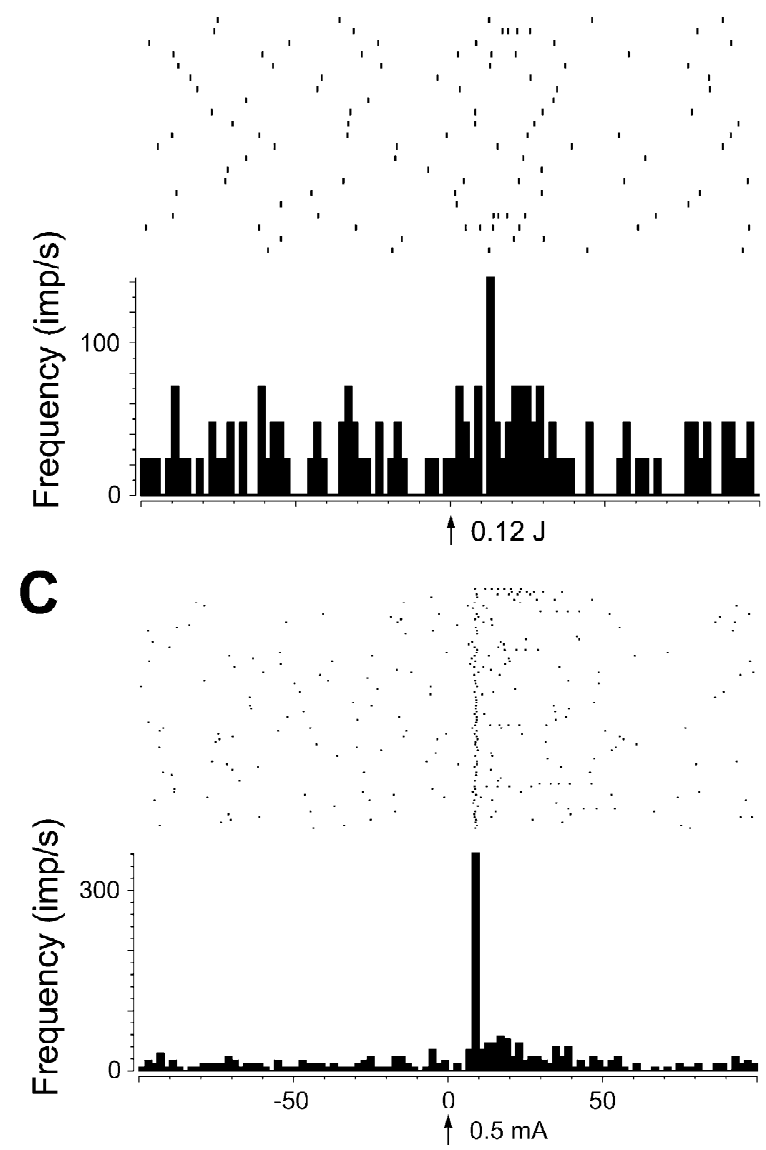

Time (ms)
B

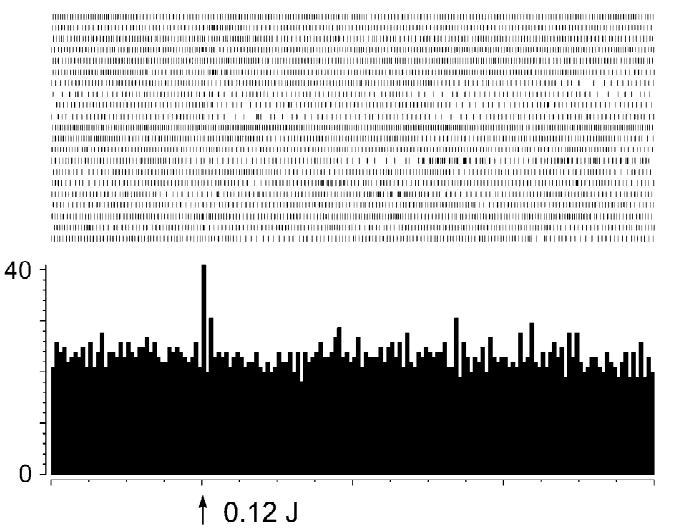

D

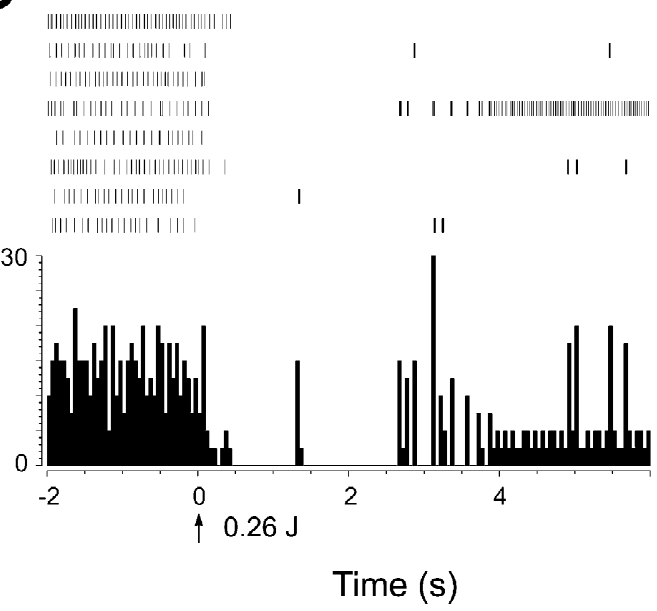

Fig. 7. (A,B) Peri-event histogram (lower panel) and dot raster (upper panel) of responses of a RT unit to laser irradiation of $12 \mathrm{~W}$, $10 \mathrm{~ms}$ of the glabrous skin of the left forepaw at time 0 of a rat under urethane anesthesia. The short latency excitatory response occurred at 12-14 ms (A). (C) Electrical stimulation $(0.5 \mathrm{~mA}$ intensity, $2 \mathrm{~ms}$ duration at $1 \mathrm{~Hz}$ ) of the paw produced consistent short-latency excitation of the same unit at 8-10 ms. (D) The same unit with laser irradiation of $13 \mathrm{~W}, 20 \mathrm{~ms}$. Note the inhibition of activity $150 \mathrm{~ms}$ after laser irradiation. Bin width: $2 \mathrm{~ms}$ for (A) and (C), $50 \mathrm{~ms}$ for (B) and (D).

\subsection{RT as a modality gate}

By concentrating on well-isolated thalamic units whose nociceptive receptive fields are easier to assess, i.e. those lying in the glabrous skin of the paws, we were able to identify two types of somatosensory RT neurons, namely, nociceptive-related RT neurons and tactile-only RT neurons. Tactile-only RT neurons were excited by innocuous tactile stimuli. When noxious stimuli were applied, they showed no further excitation or inhibition. The nociceptive-related RT neurons were also excited by innocuous tactile stimuli, but noxious stimuli inhibited this population of neurons.

Many neurons in the brain are inhibited by noxious stimulation (amygdala [2]; dorsal raphe [28]; rostral ventral medulla [13]). What makes the inhibition of a population of RT neurons by nociceptive inputs especially interesting is that the RT lies directly in the major throughway going from the VB to the somatosensory cortices. RT neurons are GABAergic and send inhibitory outputs to the thalamus. The RT might thus be performing modality gating of the somatosensory afferent inputs. Innocuous tactile inputs, e.g. light touch, will excite RT neurons, which by virtue of their negative feedback, will modulate (i.e. inhibit) subsequent tactile inputs going through the thalamocortical pathway $[20,24,25]$ (but see pages $74-75$ in [40]). On the other hand, noxious inputs will inhibit RT neurons (Figs. 3 and 8). By turning off the negative feedback, noxious inputs are permitted to transmit to the somatosensory cortex unimpeded as if an imaginary gate has been opened.

It is crucial to distinguish the postexcitatory inhibition of VB neurons produced by RT feedback versus the prolonged inhibition on RT neurons themselves as reported in the present study. Low-intensity electrical stimulation or light tactile stimuli activated thalamocortical neurons in the VB, which activates RT neurons monosynaptically. RT 


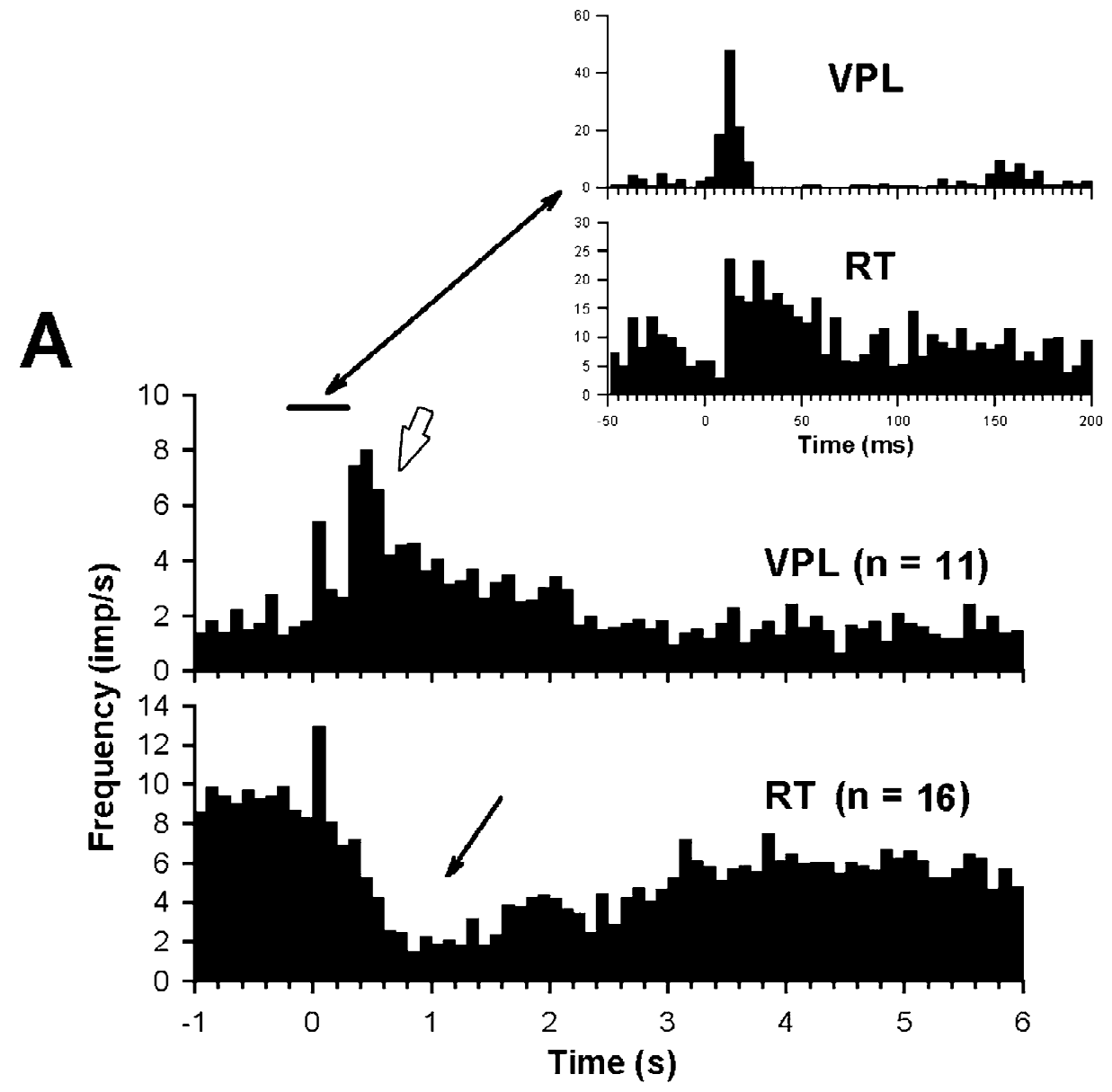

B

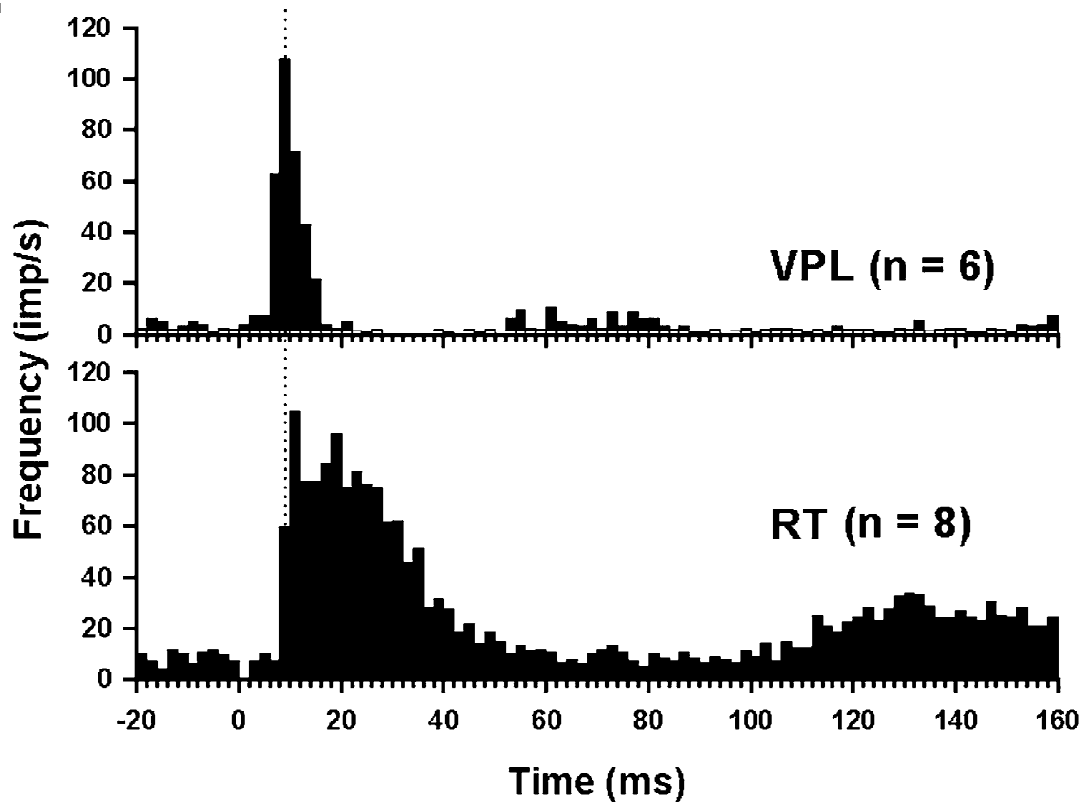

Fig. 8. (A) Averaged peri-event histogram of 16 VPL units and 11 RT units before and after laser irradiation of $10-13 \mathrm{~W}, 10-20 \mathrm{~ms}$ at time 0 . Note short-latency and long-latency (open arrow) excitations of the VPL units, and short-latency excitation and long-latency inhibition (thin arrow) of the RT units. The short-latency responses are more clearly illustrated in the expanded histogram (inset, top panel: VPL units, lower panel: RT units). Bin size is $100 \mathrm{~ms}$ ( $5 \mathrm{~ms}$ for the inset). (B) Averaged peri-event histogram of $6 \mathrm{VPL}$ units and $8 \mathrm{RT}$ units before and after a tactile stimulation of $5 \mathrm{~ms}, 10 \mathrm{~g}$ at time 0 . Note that the VPL units peaked at $10 \mathrm{~ms}$ (dotted line), slightly leading the RT peak. 
neurons, by virtue of their inhibitory synapses on VB neurons, produce a period of quiescence of thalamocortical neurons (note the postexcitatory inhibition of the VPL units from 20 to $50 \mathrm{~ms}$ in Fig. $8 \mathrm{~B}$ of the present study). Postexcitatory inhibition is one of the mechanisms by which the whisker-related RT neurons phasically modulate the receptive field and the dynamic responsiveness of VPM cells $[20,24,25]$. On the other hand, prolonged inhibition of nociceptive-related RT neurons observed here may make possible the very-high-frequency discharge of nociceptiveprocessing VPL neurons following a noxious stimulus. The high-frequency discharge of these VPL (or other somatosensory thalamic nuclei) neurons may be important in the higher-level processing of pain information and crucial for alerting the animal to impending danger.

The proposed function of the RT modality gate may be an integral part of the 'pain control mechanism'. Indeed, the nociceptive-related RT neuron behaves somewhat like the OFF cell described in the medullospinal circuitry $[12,13]$. For example, the discharge of an OFF cell is terminated by noxious stimulation. Other properties of OFF cells, such as activation by morphine, and activity related to suppression of nociceptive transmission, have yet to be tested for the RT system. It is certain, however, that operation of nociceptive-related RT neurons is restricted only to the thalamic level, judging by the known terminal distribution of the RT axon. In this aspect, it would be interesting to study whether the tactile-only and the nociceptive-related RT neuron types found in the present study have differential axonal terminal patterns in the thalamus [7].

We tested RT neurons under four types of general anesthetics, namely, pentobarbital, urethane, ketamine and halothane. In addition to demonstrating that nociceptive inputs had a general inhibitory effect on RT neurons in anesthetized condition, the result that nociceptive inhibition on RT was stronger in rats under GABAergic potentiating pentobarbital than that under non-GABAergic urethane and ketamine [32] has very interesting implications. This indicates that in situations where the GABAergic mechanism is activated endogenously, for instances, during drowsiness or slow-wave sleep [14], RT might be under stronger inhibitory influence of peripheral nociceptive inputs. Thus, during the period of slow-wave sleep, innocuous tactile inputs will be gated, whereas the body would be more quickly alerted by a noxious stimulus if RT neurons would not impede flow of noxious signals to the cortex.

Mantagne-Clavel and Oliveras [29] recorded about 21 RT units in awake, tethered rats. They found four widedynamic range type units responded to noxious pinching. Because inhibition was not mentioned, and the same units were not tested under anesthetized conditions, it is unclear how these units relate to the two types of RT neurons reported here. It is likely that units classified under anesthesia preparation might change their responsive pattern in a wakeful condition [5]. Whether the 'modality gate' proposed in the present study is a conditional gate, which operates only under a heightened GABAergic condition, remains to be tested systematically in a more natural setting.

\subsection{Possible mechanism of $R T$ inhibition by nociceptive input}

Since thalamocortical neurons monosynaptically excite RT neurons, what could be the source of this inhibition by nociceptive input? As shown in Table 2, after the removal of the ipsilateral sensorimotor cortex, most of the RT neurons tested were still inhibited by noxious stimuli. This indicates that inhibition of RT neurons might not depend on the cortical circuit.

Thalamocortical cells in the VPL show periods of inhibition following afferent stimulation [49]. That postexcitatory inhibition following the short-latency excitatory response is not the major cause here can be clearly seen from Figs. 6A and 7D. In either case, there was no excitation preceding the long-latency inhibition.

There might be three possible mechanisms responsible for the inhibition of RT neurons by nociceptive inputs, and these mechanisms are not mutually exclusive. In a preliminary study [47], we tested the effects of noxious stimulation of a heterotopic limb. The RT units had an excitatory tactile receptive field in the forepaw and was inhibited by pinching the homotopic forepaw. Furthermore, in half of the twelve units tested, the same unit was also inhibited by pinching of the hindpaw. Clearly, RT neurons have a larger inhibitory nociceptive field than excitatory tactile field. Noxious stimuli, whether mechanic or thermal, will activate a larger area, thereby more strongly activating the inhibitory nociceptive field.

There are abundant intra-RT connections between adjacent RT neurons [8,36,48]. These connections can be dendro-dendritic or axo-dendritic. By their GABAergic nature, these intra-RT connections are mostly inhibitory. When a part of the VPL is strongly activated by a nociceptive input, it is possible that the directly connected RT part will be activated first, but soon afterwards, intraRT inhibition may dominate and suppress most surrounding RT activities.

The third possible mechanism is that RT activity might be turned off by its inhibitory brainstem inputs $[4,9,43]$. These inputs may include cholinergic ones from basal forebrain and parabrachial regions [26,35].

In summary, we have shown single-unit evidence that many RT neurons are inhibited by nociceptive inputs in anesthetized rats. How this inhibition is used in the somatosensory function and what possible role this plays in the daily life of the rat await further studies in conscious and behaving animals. 


\section{Acknowledgements}

We thank Dr. R.C.S. Lin for helpful comments. Supported by grants NSC89-2311-b002-039 and NHRI-EX909018EP

\section{References}

[1] P. Barbaresi, R. Spreafico, C. Frassoni, A. Rustioni, GABAergic neurons are present in the dorsal column nuclei but not in the ventroposterior complex of rats, Brain Res. 382 (1986) 305-326.

[2] J.F. Bernard, G.F. Huang, J.M. Besson, Nucleus centralis of the amygdala and the globus pallidus ventralis: electrophysiological evidence for an involvement in pain process, J. Neurophysiol. 68 (1992) 551-569.

[3] F. Block, Stimulation of $N$-methyl-D-asparate receptors in the rat nucleus reticularis thalami suppresses somatosensory evoked potentials, Brain Res. 636 (1994) 143-146.

[4] E. Carstens, J. Leah, J. Lechner, M. Zimmermann, Demonstration of extensive brainstem projections to medial and lateral thalamus and hypothalamus in the rat, Neuroscience 35 (1990) 609-626.

[5] J.K. Chapin, B.D. Waterhouse, D.J. Woodward, Differences in cutaneous sensory response properties of single somatosensory cortical neurons in awake and halothane-anesthetized rats, Brain Res. Bull. 6 (1981) 63-70.

[6] A.D. Craig, J.O. Dostrovsky, Medulla to thalamus, in: P.D. Wall, R. Melzack (Eds.), Textbook of Pain, 4th Edition, Churchill Livingstone, Edinburgh, 1999, pp. 183-214.

[7] C.L. Cox, J.R. Huguenard, D.A. Prince, Heterogeneous axonal arborizations of rat thalamic reticular neurons in the ventrobasal nucleus, J. Comp. Neurol. 366 (1996) 416-430.

[8] M. Deschenes, A. Madariaga-Domich, M. Steriade, Dendrodendritic synapses in the cat reticularis thalami nucleus: a structural basis for thalamic spindle synchronization, Brain Res. 334 (1985) 165-168.

[9] R. Dingledine, J.S. Kelly, Brain stem stimulation and the acetylcholine-evoked inhibition of neurons in the feline nucleus reticularis thalami, J. Physiol. (Lond.) 271 (1977) 135-154.

[10] L. Domich, G. Oakson, M. Steriade, Thalamic burst patterns in the naturally sleeping cat: a comparison between cortically projecting and reticularis neurons, J. Physiol. 379 (1986) 429-449.

[11] R.J. Fan, B.C. Shyu, S. Hsiao, Analysis of nocifensive behavior induced in rats by $\mathrm{CO}_{2}$ laser pulse stimulation, Physiol. Behav. 57 (1995) 1131-1137.

[12] H.L. Fields, A.I. Basbaum, Central nervous system mechanisms of pain modulation, in: P.D. Wall, R. Melzack (Eds.), Textbook of Pain, 4th Edition, Churchill Livingstone, Edinburgh, 1999, pp. 309-329.

[13] H.L. Fields, J. Bry, I.D. Hentall, G. Zorman, The activity of neurons in the rostral medulla of the rat during withdrawal from noxious heat, J. Neurosci. 3 (1983) 2545-2552.

[14] C. Gottesmann, GABA mechanisms and sleep, Neuroscience 111 (2002) 231-239.

[15] G. Guilbaud, M. Pechanski, M. Gautron, D. Binder, Neurons responding to noxious stimulation in VB complex and caudal adjacent regions in the thalamus of the rat, Pain 8 (1980) 303-318.

[16] G. Guilbaud, V. Kayser, J.M. Benoist, M. Gautron, Modifications in the responsiveness of rat ventrobasal thalamic neurons at different stages of carrageenin-produced inflammation, Brain Res. 385 (1986) $86-98$.

[17] G. Guilbaud, V. Kayser, The ventrobasal complex of the rat thalamus: its involvement in the sensory-discriminative aspects of nociception, including models of clinical pain, in: J.M. Besson, G. Guilbaud, M. Pechanski (Eds.), Thalamus and Pain, Elsevier, 1987, pp. $185-209$.
[18] R.W. Guillery, S.L. Feig, D.A. Lpzsadi, Paying attention to the thalamic reticular nucleus, Trends Neurosci. 21 (1998) 28-32.

[19] R.M. Harris, A.E. Hendrickson, Local circuit neurons in the rat ventrobasal thalamus-a GABA immunocytochemical study, Neuroscience 21 (1987) 229-236.

[20] J.A. Hartings, S. Temereanca, D.J. Simons, High responsiveness and direction sensitivity of neurons in the rat thalamic reticular nucleus to vibrissa deflections, J. Neurophysiol. 83 (2000) 2791-2801.

[21] C.N. Honda, E.R. Perl, S. Mense, Neurons in ventrobasal region of cat thalamus selectively responsive to noxious mechanical stimulation, J. Neurophysiol. 49 (1983) 662-673.

[22] E.G. Jones, The Thalamus, Plenum Press, New York, 1985.

[23] N. Koyama, Y. Nishikawa, J. Chen Jr., R.P. Barderrama, T. Yokota, Differential inhibitory mechanisms in VPL versus intralaminar nociceptive neurons of the cat: II. effects of systemic morphine and CCK, Jpn. J. Physiol. 45 (1995) 1029-1041.

[24] S.M. Lee, M.H. Friedberg, F.F. Ebner, The role of GABA-mediated inhibition in the rat ventral posterior medial thalamus. I. Assessment of receptive field changes following thalamic reticular nucleus lesions, J. Neurophysiol. 71 (1994) 1702-1715.

[25] S.M. Lee, M.H. Friedberg, F.F. Ebner, The role of GABA-mediated inhibition in the rat ventral posterior medial thalamus. II. Different effects of $\mathrm{GABA}_{\mathrm{A}}$ and $\mathrm{GABA}_{\mathrm{B}}$ receptor antagonists on responses of VPM neurons, J. Neurophysiol. 71 (1994) 1716-1726.

[26] D.A. McCormick, Cholinergic and noradrenergic modulation of thalamocortical processing, Trends Neurosci. 12 (1989) 215-221.

[27] D. Mitchell, R.F. Hellon, Neuronal and behavioral responses in rats during noxious stimulation of the tail, Proc. R. Soc. Lond. B 197 (1977) 169-194.

[28] J. Montagne-Clavel, J.L. Oliveras, G. Martin, Single-unit recordings at dorsal raphe nucleus in the awake-anesthetized rat: spontaneous activity and responses to cutaneous innocuous and noxious stimulations, Pain 60 (1995) 303-310.

[29] J. Montagne-Clavel, J.L. Oliveras, Does barbituate anesthesia modify the neuronal properties of the somatosensory thalamus? A single-unit study related to nociception in the awake-pentobarbital treated rat, Neurosci. Lett. 196 (1995) 69-72.

[30] V.M. Montero, Amblyopia decreases activation of the corticogeniculate pathway and visual thalamic reticularis in attentive rats: a 'focal attention' hypothesis, Neuroscience 91 (1999) 805-817.

[31] S. Mushiake, A. Shosaku, Y. Kayama, Inhibition of thalamic ventrobasal complex neurons by glutamate infusion into the reticular nucleus in rats, J. Neurosci. Res. 12 (1984) 93-100.

[32] L.E. Nelson, T.Z. Guo, J. Lu, C.B. Saper, N.P. Franks, M. Maze, The sedative component of anesthesia is mediated by GABAA receptors in an endogenous sleep pathway, Nature Neurosci. 5 (2002) 979-984.

[33] M. Pechanski, G. Guilbaud, M. Gautron, Neuronal responses to cutaneous electrical and noxious mechanical stimuli in the nucleus reticularis thalami of the rat, Neurosci. Lett. 20 (1980) 165-170.

[34] M. Pechanski, G. Guilbaud, M. Gautron, J.M. Besson, Encoding of noxious heat messages in neurons of the ventrobasal thalamic complex of the rat, Brain Res. 197 (1980) 401-413.

[35] D. Pinault, M. Deschenes, Muscarinic inhibition of reticular thalamic cells by basal forebrain neurons, NeuroReport 3 (1992) 1101-1104.

[36] D. Pinault, Y. Smith, M. Deschenes, Dendrodendritic and axoaxonic synapses in the thalamic reticular nucleus of the adult rat, J. Neurosci. 17 (1997) 3215-3233.

[37] C. Reyes-Vazquez, N. Dafny, The parafasciculus thalami as a site for mediating the antinociceptive response to GABAergic drugs, Brain Res. 383 (1986) 177-184.

[38] F.-Z. Shaw, R.-F. Chen, H.-W. Tsao, C.-T. Yen, Comparison of touch- and laser heat-evoked cortical field potentials in conscious rats, Brain Res. 824 (1999) 183-196.

[39] F.-Z. Shaw, R.-F. Chen, C.-T. Yen, Dynamic changes of touch- and laser heat-evoked field potentials of primary somatosensory cortex 
in awake and pentobarbital anesthetized rats, Brain Res. 911 (2001) $105-115$.

[40] S.M. Sherman, R.W. Guillery, Exploring the Thalamus, Academic Press, San Diego, 2001.

[41] A. Shosaku, Y. Kayama, I. Sumitomo, Somatotopical organization in the rat thalamic reticular nucleus, Brain Res. 311 (1984) 57-64.

[42] A. Shosaku, Y. Kayama, I. Sumitomo, M. Sugitani, K. Iwama, Analysis of recurrent inhibitory circuit in rat thalamus: neurophysiology of the thalamic reticular nucleus, Prog. Neurobiol. 32 (1989) 77-102.

[43] M.W. Sofroniew, V.J. Priestley, A. Consolazione, F. Eckenstein, A.C. Cuello, Cholinergic projections from the midbrain and pons to the thalamus in the rat, identified by combined retrograde tracing and choline acetyltransferase immunohistochemistry, Brain Res. 329 (1985) 213-223.

[44] M. Steriade, R.R. Llinás, The functional states of the thalamus and the associated neuronal interplay, Physiol. Rev. 68 (1988) 649-742.

[45] M. Steriade, E.G. Jones, D.A. McCromick, in: Thalamus. Organization and Function, Vol. 1, Elsevier, Oxford, Amsterdam, 1997.
[46] W.D. Willis, The Pain System: the Neural Basis of Nociceptive Transmission in the Mammalian Nervous System, 1985.

[47] C.-T. Yen, Inhibition of thalamic reticular activities by nociceptive input in the rat, Abstr. Soc. Neurosci. 26 (2000) 432.

[48] C.-T. Yen, M. Conley, S.H.C. Hendry, E.G. Jones, The morphology of physiologically identified GABAergic neurons in the somatic sensory part of the thalamic reticular nucleus in the cat, J. Neurosci. 5 (1985) 2254-2268.

[49] C.-T. Yen, M. Conley, E.G. Jones, Morphological and functional types of neurons in cat ventral posterior thalamic nucleus, J. Neurosci. 5 (1985) 1316-1338.

[50] C.-T. Yen, T.-C. Fu, R.-C. Chen, Distribution of thalamic nociceptive neurons activated from the tail of the rat, Brain Res. 498 (1989) $118-122$.

[51] C.-T. Yen, C.-H. Huang, S.-E. Fu, Surface temperature change, cortical evoked potential and pain behavior elicited by $\mathrm{CO}_{2}$ lasers, Chin. J. Physiol. 37 (1994) 193-199. 